\title{
Development of a Universal Waste Management System
}

\author{
Thomas J. Stapleton ${ }^{1}$ \\ United Technologies Aerospace Systems, Windsor Locks, Connecticut, 06096-1010
}

\author{
Shelley Baccus ${ }^{2}$, James L. Broyan, Jr. ${ }^{3}$ \\ Crew Habitation Hardware \& Technology Development, NASA Johnston Space Center, Houston, Texas, 77598
}

\begin{abstract}
NASA is working with a number of commercial companies to develop the next low Earth orbit spacecraft. The hardware volume and weight constraints are similar to or greater than those of the Apollo era. This, coupled with the equally demanding cost challenge of the proposed commercial vehicles, causes much of the Environmental Control and Life Support System (ECLSS) designs to be reconsidered. The Waste Collection System (WCS) is within this group of ECLSS hardware. The development to support this new initiative is discussed within. A WCS concept - intended to be common for all the vehicle platforms currently on the drawing board - is being developed. The new concept, referred to as the Universal Waste Management System (UWMS), includes favorable features from previous designs while improving on other areas on previous Space Shuttle and the existing International Space Station (ISS) WCS hardware, as needed. The intent is to build a commode that requires less crew time, improved cleanliness, and a $75 \%$ reduction in volume and weight compared to the previous US ISS/Extended Duration Orbitor WCS developed in the 1990s. The UWMS is most similar to the ISS Development Test Objective (DTO) WCS design. It is understood that the most dramatic cost reduction opportunity occurs at the beginning of the design process. To realize this opportunity, the cost of each similar component between the UWMS and the DTO WCS was determined. The comparison outlined were the design changes that would result with the greatest impact. The changes resulted in simplifying the approach or eliminating components completely. This initial UWMS paper will describe the system layout approach and a few key features of major components. Future papers will describe the UWMS functionality, test results, and components as they are developed.
\end{abstract}

\section{Nomenclature}

ACV = Ассенизацио нно- санитарное устройство roughly translated as the Russian Waste Management System. For purpose of this paper it is equivalent to the US acronym WCS

$C E V=$ Crew Exploration Vehicle

DTO $=$ Development Test Objective

ECLSS = Environmental Controls and Life Support Systems

$I S S=$ International Space Station

MMSEV $=$ Multi-Mission Space Exploration Vehicle

UWMS = Universal Waste Management System

WCS $=$ Waste Collector System

\footnotetext{
${ }^{1}$ Universal Waste Management System Co-Investigator, United Technologies Aerospace Systems, One Hamilton Road, Windsor Locks, CT, 06096, 77058/Mail Stop EC7, not AIAA affiliated.

${ }^{2}$ AES Logistics Reduction and Repurposing Project Manager, Crew \& Thermal Systems Division, 2101 NASA

Parkway, Houston, TX, 77058/Mail Stop EC7, not AIAA affiliated.

${ }^{3}$ AES Logistics Reduction and Repurposing SE\&I Lead, Crew \& Thermal Systems Division, 2101 NASA Parkway, Houston, TX, 77058/Mail Stop EC2, not AIAA affiliated.
}

\section{Introduction}


$\mathrm{T}^{\mathrm{h}}$ he development of human metabolic waste collection for spacecraft presents some significant challenges without the presence of gravity. Terrestrial and aircraft commode systems rely on gravity and/or vacuum assist but are not applicable to space flight. In particular, the separation and capture of fecal and urine waste from the crew members can only be rudimentarily tested on the ground or with the approximately 20 seconds of reduced gravity on a parabolic flight aircraft. Additionally, water is a valuable resource in space flight and necessitates the separate collection of urine to allow downstream water recovery in many longer-mission durations. The history of the spacecraft Waste Collector System (WCS) (Fig. 1) development has been previously documented, ${ }^{1}$ but it is beneficial to briefly review the recent hardware systems. It is critical that future systems heavily leverage off the successful crew interfaces of previous systems because of the difficulty of validating new separation and capture techniques. ${ }^{1}$

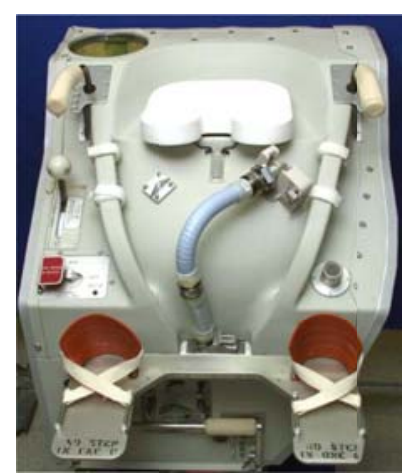

Figure 1. Shuttle WCS.

\section{Recent Waste Collector System History}

The basic Space Shuttle and International Space Station (ISS) WCS (Fig. 2) operation is to use of airflow to entrain waste. Urine is collected in a funnel held in close proximity to the Urethra. If the funnel is pressed against the body, the airflow is blocked and urine is not effectively separated from the body but instead pools in the funnel

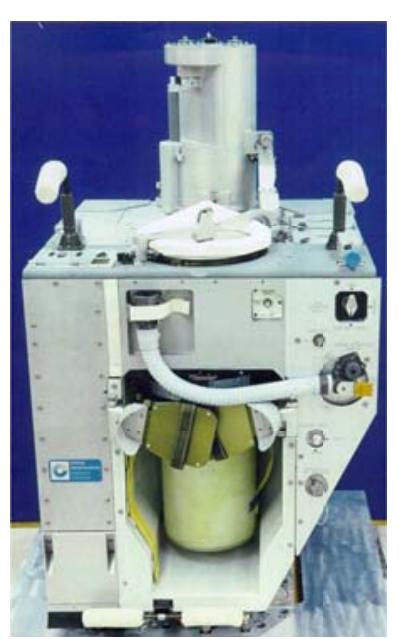

Figure 2. US ISS WCS. and hose. A variety of urinal funnels have tangential holes to allow contact with the body but allow in airflow for separation. Fecal material is collected separately by airflow entrainment. However, crew contact with a seat is preferred to facilate controlled and stable airflow. When the crew member's buttocks are in contact with the seat, air enters in a fairly uniform radial direction under the seat. Air drag then helps separate the fecal bolus and draw it into a tank or fecal collection bag. Air is separated from the urine and fecal material, treated for odors, and returned to the spacecraft cabin. Conceptually simple, the hardware implementation is highly dependent on the details to enable successful crew use. Successful capture and ease of use for simultaneous urination and defecation for mixed gender crews have been consistent limitations of the Space Shuttle and ISS WCS. Additionally, these systems are sensitive to crew alignment on the seat and can result in inadvertent fouling of the collection hardware with fecal material. The Universal Waste Management System (UWMS) plans to address these two limitations as well and improve other operations.

The Space Shuttle WCS used similar collection airflow techniques. It collected and freeze dried the fecal matter and plumbed urine to a venting station after the fecal matter was separated from the entrained air. The system worked well; however, the large, fixed fecal storage tank limited its application beyond its 16-day flight usage.

As found in an investigation by the Medical Branch at NASA Johnson Space Center, astronauts tend to drink less water in orbit. There is also a tendency for them to have more calcium and uric acid present in their urine. These tendencies work in concert and result in a higher instance of the formation of calcium and uric acid-based kidney stones. As these solids precipitate out of solution within the WCS, they tend to collect in small areas, such as the separator pitot tube, on filters, and in small valve passages. These deposits block the flow of urine and the function of included components. To combat this occurrence, urine pretreat - Oxone ${ }^{\circledR}$ - was added to lower the urine $\mathrm{pH}$ and reduce these mineral deposits from forming.

United Technologies Aerospace Systems developed the US ISS WCS with NASA in the early 1990s. The US ISS WCS shared many features of the Space Shuttle WCS, but modified the fecal collection from a large storage tank to a removable fecal canister. Using plastic lids, the compactor compressed each individual hydrophobic fecal bag to reduce storage volume. After about 21 uses, the canisters are removed, sealed with a charcoal filled lid, and stored. To improve airflow predictability, a fan was included for the urine separator, and a separate fan was provided for fecal collection.

The Russian Soyuz toilet (Soyuz ACY) (Fig. 3) is smaller than the two WCS units, and is used by the crew positioned in the aisle. It pulls cabin air through the urine funnel and fecal collector before passing through a static separator, where the urine is stored. The saturated air travels through an odor filtration before the urine and cabin air 
are separated by a centrifugal unit. The fecal collection bag is only good for one use, and the urine tank is limited to 10.2 liters. Urine collection is considered acceptable, whereas fecal collection did not receive favorable review.

The Russian Module toilet (SM ACY) (Fig. 4) has provided long-term WCS use since the ISS was first inhabited. It uses air to assist fecal and urine collection. A centrifugal urine separator removes air from the urine stream. The air flows to a charcoal filter prior to reentry back into the cabin. This unit has an air ring under the seat, similar to the two American designs discussed, to control odor and assist in fecal capture. The fecal canister holds one bag that is pushed into a collection tank after each use. This tank can hold about 21 defecations before changing out. The urine is pretreated with a mixture of deionized water, sulfuric acid, and chromium trioxide to reduce the $\mathrm{pH}$ to 1.3 to 2.0 . $^{2}$

The commode is one of the most intimate systems that are flown. Crew consensus is paramount regarding which elements are needed, or which need improvement. From review of the previous solutions. the desired elements include:

- Adequate air flow for urine and fecal collection

- A seat configuration that lends to ease of use and maintaining cleanliness

- Urine air separation, traditionally performed by centrifugal separators

- A flexible urinal hose and funnel integration for male and female crew

- Clean and easy fecal collection that supports long-term missions

- Stowing urine on-board supports water recovery

- Overall user simplicity

\section{Universal Waste Management System Development}

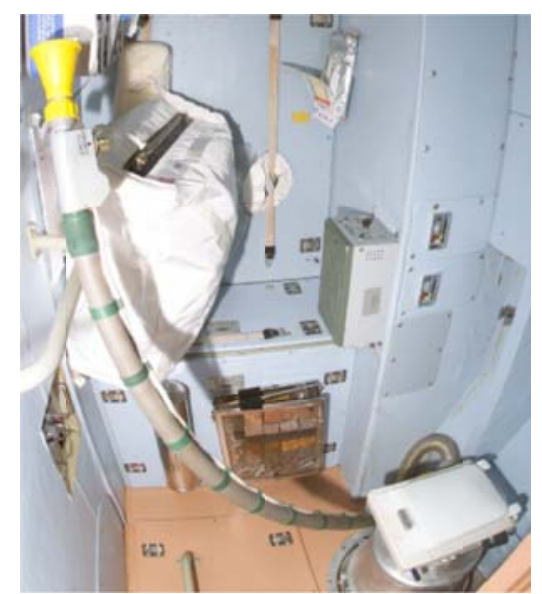

Figure 4. SM ACY.

At the initiation of creating a new WCS, the design team attempted to capture all of the elements and features that had proven favorable in the previous designs and enhance on features that had been identified as needing improvement. These goals were to be completed while coupling all the required features into a system that was considerably smaller, lighter, and less expensive that its predecessors. As a design goal to reduce recurring costs, the team developed the UWMS as a cross platform solution. The design is intended to have a central set of common hardware, and include the ability to be modified so that different permeations may be offered to different space ship and space station designers. The modifications may include pluming urine directly to a water processing unit or having greater-capacity fecal canisters, for instance.

To complete the goal identified, engineers performed an in-depth review of all previous systems, including literature searches of internal and publically available text, reviewing crew consensus, and speaking with experts within the NASA community. While trying to make sense of all the compiled data, the engineers used the ISS DTO WCS as the baseline, and documented the costs for all of the included components. They then performed a true costto-value-added comparison. This effort identified the opportunities needed to influence the overall design to meet the performance and cost improvements. The resultant design modifications are outlined below.

The UWMS was constructed as two individual systems - one for fecal collection and the other for urine collection. This enhances the ability for design flexibility. With this distinction, the frame found in traditional systems was removed and replaced by a simple monocoque frame that was designed to house all of the elements within the UWMS, as can be seen in Fig. 6. In this section, each of these components included in these subsystems will be discussed regarding what was taken from previous designs and what we modified, added, or deleted.

\section{A. Fecal Collection}

As found in the US ISS WCS, fecal matter is collected and stored in a removable canister. Odor control and clean collection of fecal matter have proven central. This section will discuss the different elements that are included to satisfy this goal 


\section{B. Seat, Lid, and Air Ring}

The UWMS seat (Fig. 5) is similar to the US ISS WCS. It offers smooth surfaces for cleanliness, includes a tailbone section to encourage crew cheek separation, and has an integrated air ring to aid in odor control and bolus separation from the crew. In microgravity, it is difficult for the crew to locate themselves properly on the seat. To assist in this operation, the through diameter was reduced and small ridges where added to help them find the "home" position. Another recurring WCS problem has been that the female crews have trouble positioning the urine funnel properly while simultaneously defecating. To enable simultaneous use, the seat was moved forward from its central location, allowing the funnel to be moved closer female crew member's body, and to be positioned a more favorable upright angle. The cutaway

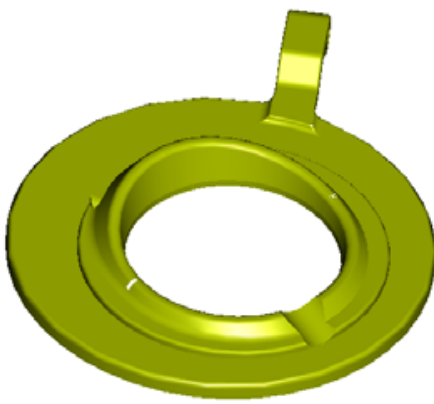

Figure 5. Seat. section was included from previous designs to further assist in proper funnel location.

The lid was changed from a "plug" design to an external cover. This change removed a sealing seat that interrupted the through diameter in the US ISS WCS design, which improves cleanliness. This change replaced a complex cam latching system with a simple over-center latch that may be purchased as an off-the-shelf hardware component to reduce overall costs.

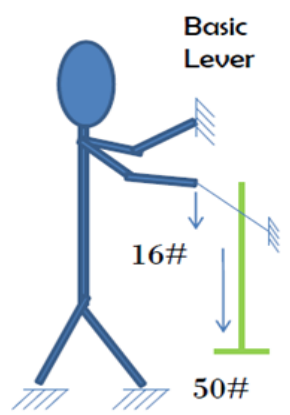

Figure 6. Manual compactor.

\section{Fecal Collection}

The UWMS Fecal Collection system is very similar to the ISS DTO WCS, which received favorable crew consensus regarding its cleanliness. A single, hydrophobic bag is placed under the hinged seat; upon completion a formed, previously qualified lid is placed on the bag.

The ISS DTO WCS used a motorized fecal compactor to collect and compress the bag and lid, and the included waste. The design was large, heavy, and expensive, and required excessive crew time to operate. To reduce volume and crew operation, the team developed a simple lever mechanism that allowed the crew to manually compress the waste. As shown in Fig. 6, 16 pounds of crew force is multiplied by the mechanical advantage to supply 50 pounds of force. This downward force is required to ensure that the waste stowing volume is minimized to improve overall volume efficiency. The manual compactor was designed to fold up to allow for easy stowage.

The fecal canister is similar to the ISS DTO WCS version, and includes ridges that prohibit the captured waste from expanding (referred to as "spring back"). The previous capturing system was replaced with an over-center latch that is identical to the one found on the new seat lid. This modification reduces both hardware costs and the amount of crew time required to remove and replace the canister.

\section{Odor/Bacteria Filter}

Air is pulled from the commode and delivered to an odor/bacteria filter to capture odor-causing molecules and bacteria. It consists of activated charcoal developed for use on the ISS WCS. Previous odor/bacteria filters were cylinder in form and were housed separately. To reduce system volume, the UWMS filter surrounds the commode transfer tube (the conduit between the seat and fecal collection canister). The filter will be developed as an Orbital Replacement Unit to support longer-duration missions (in excess of 210 crew days).

\section{E. Crew Restraint System}

Thigh bars and foot restraints, included in both American systems, helped the crew stay secure and positioned properly in the microgravity environment. Although effective, they were considered to be more complex than needed. The SM ACY uses a toe bar mounted to the floor directly in front of the crew. This was reported as a favorable design, it allowed the crew to secure their feet under the Toe Bar and apply torque to their body as a controlling method. This form of restraint will be included as the UWMS restraint system. 


\section{F. Urine Storage System}

The American commodes (Space Shuttle WCS and ISS DTO WCS) both vented urine overboard, which required a storage tank, valves, and heated vent orifices. Following extensive trade studies, it was decided that the UWMS should store the urine on board. This approach removes the complex venting systems and allows the urine to be processed in station applications, recalling that the vented urine also accounts for about $80 \%$ of water consumed by the crew.

In the UWMS a urine fan will pull air from the funnel and through the hose (both components are identical to the US ISS WCS), and into the centrifugal separator. The separator will pump urine into a bag within a rigid cylinder. An internal sensor at the base of the rigid enclosure senses when the bag is full, and directs a three-way valve to redirect the flow to a second flexible bag. The sensor will also send a signal that turns on an indication light, alerting the crew to change out the filled bag. Each bag will house about 12 uses. The crew will be responsible for removing the urine bag in low Earth orbit vehicles. For station application, the urine may plumb directly to the Urine Processing Unit.

\section{G. Dual Fan Separator}

The ISS DTO WCS included a urine separator fan, a commode fan, and a centrifigul air/urine separator. Previous design would require three motors and three motor controllers to power the rotating equipment combination.Coupled with associated harnessing, supporting structure, and internal plumbing, the previous designs resulted in a large, heavy, and expensive solution. The UWMS combines these three functions onto a single shaft, known as the dual fan separator. This device rotates at a single operating speed, supplying air flow to the urinal hose and seat air ring. The included urine separator was chosen from the Space Shuttle WCS. It has been developed and used for the majority of all shuttle flights. Design changes are currently being considered to improve maintenance of the urine separator.

\section{H. User Panel}

General crew consensus is that the user panels on previous sytems are complex, offering more functioality than is really needed. Instead of a user panel, the UWMS will turn on when the seat lid is lifted 2 degrees. When the lid is opened, the dual fan separator will be fully operative, supplying air flow to the seat and urinal hose. The urine separator will also be operating. The dual fan separator will continue to operate for about 30 seconds after the lid is closed to ensure that all urine is processed.

Each urine tank has a signal light. When the internal sensor determines the urine bag is full, the lights will illuminate. A second visual indicator is found on the fecal canisters to instruct that the canister is full and ready to be replaced.

\section{Summary}

The UWMS was developed to capture the design and operation elements that resulted from years of WCS development and flight experience. A mock-up of the UWMS was built and sent to Johnston Space Center for review. Currently, the design team is working closely with NASA to develop the commode for use within the Gen 2B Multi-Mission Space Exploration Vehicle (MMSEV). This ground unit is considered the Gen 3 UWMS and will be fully functional. The Gen 3 Unit is intended to support the MMSEV manned testing and to act as a tool allowing crew an early opportunity to influence development of the universal system.

The main goal is to work with all stakeholders early in the design so that, upon design completion, it will be readily accepted into the low Earth orbit ships being designed for the Commercial Crew Program, the MMSEV, and the Orion Crew Exploration Vehicle. This effort is beneficial for three main reasons:

- Developing a universal configuration allows the non-recurring costs to be divided among many applications

- The time being spent allows for a commode that is considerably smaller and lighter than the predecessors that shared the full feature compliment

- The simplicity of the design and removal of expensive components allow the UWMS to compete in the new era of commercial space travel 


\section{References}

${ }^{1}$ Broyan, J. L., “CEV Waste Collector System Technology Comparisons for Constellation Applications,” 2007-01-3227, 37 International Conference on Environmental Systems, Chicago, 2007. , 12 January 2006. 